
\title{
Surveillance \& Society
surveillance in contemporary health and
social care: friend or foe?
}

\section{Heather Morgan}

University of Aberdeen, UK.

h.morgan@abdn.ac.uk

\begin{abstract}
This research note concerns the increasing ranges of technologies that are being used to monitor health and wellbeing. Many of these can be broadly interpreted as 'surveillant', implying powerful watching, even if this is not widely acknowledged. However, surveillance entails connotations of potentially inappropriate intrusion and impairment of valued freedoms. Therefore, the social and ethical ramifications of surveillance for health are a concern, particularly as technological advances are continually overtaking knowledge and understanding of them. While surveillance in health-related contexts has received some attention, conceptual and practical issues and social and ethical implications continue to arise with new, diverse and rapidly proliferating technologies. These need further investigation, especially because the direction of surveillance can be either health professional-led ('surveillance'), or patient- or group-led ('sousveillance'). This distinction has recently been identified as relevant to the research gap, particularly in respect of everyday life settings and integration, the 'domestication', of healthcare technologies. Ongoing and proposed future research explores social and ethical considerations pertaining to surveillant technologies for health and social care in the context of people's everyday lives to contribute greater knowledge and understanding of health surveillance and its complexities.
\end{abstract}

\section{Introduction}

Vast and diverse technologies are increasingly being integrated into contemporary healthcare contexts, often in spite of their infancy. Many of them can be considered surveillant, although they are not widely identified as such. National governments and agencies are increasing emphasis on these technologies. They include digital and mobile devices to capture and relay measurements for clinical review and systems for early intervention and self-management. Additionally, personal alarms, devices and sensors in the home, which monitor daily activity patterns to facilitate medication prompting, are supported. However, little is known about individual and group perceptions and experiences, particularly of these technologies as surveillant. This lack of knowledge about perceptions of these technologies, and that actors are not necessarily cognisant of their possibility as surveillant, might constitute a dangerous present and an even more dangerous future: Britain was once described as 'sleepwalking' into a surveillance society (Information Commissioner 2006) and contemporary surveillance is neither limited nor specific in scope or design (Lyon 2002). As such, there are social and ethical implications pertaining to greater reliance on health and social care delivered through surveillant means. There is, therefore, a need to critically investigate and develop knowledge of the implications of surveillant technologies for everyday health and wellbeing of individuals and groups experiencing them-as friend or foe-to inform appropriate policies and practices for their future use and regulation.

'Surveillance' is a contentious practice: it is commonly considered dangerous and potentially harmful. The 
Oxford English Dictionary defines surveillance as: 'Watch or guard kept over a person, etc., esp. over a suspected person, a prisoner, or the like; often, spying, supervision; less commonly, supervision for the purpose of direction or control, superintendence (n.)'. Thus, the critical academic investigation of surveillance is largely contained within legal and socio-political discourses of security/privacy and governance/resilience, traditionally applied within criminological and civil liberties frameworks. However, various forms of surveillance permeate ordinary everyday life (Morgan 2013) and surveillance also has been problematised in the context of health (see Critical Public Health 2013; Surveillance \& Society 2009). This has been specifically accomplished through the application of Foucault's 'extended medical gaze', promoting 'a regime of total health' in which 'the individual is not just subjected to the technologies of medical surveillance, but is expected to engage in the practice of self-surveillance' (Earle et al. 2009: 96). This acknowledgement recognises a shift from the possibility to a reality of mass surveillance in health and redistribution of power where a more active role for the patient is necessitated.

Nevertheless, scholarly approaches to health and wellbeing have focused on traditional constructions of 'surveillance' using vernaculars of authority, suppression, restriction and obligation: control rather than care. This resonates with Foucault's 'medical gaze': people's minds and bodies are separated through biological reductionism and the body becomes an object and potential target for manipulation (1973 [1963]), for example through the technologies themselves, through clinicians' uses of the technologies and also through the applied technologically-oriented research that these technologies engender (e.g. International Journal of Monitoring and Surveillance Technologies Research 2013). The implication is that Foucault's conceptualisation of the modernised empirical biomedical model (generally considered to have advanced knowledge through which models of shared decision making and supporting selfmanagement have emerged) renders the notion of surveillance for health and wellbeing paradoxical. Tensions arise between being observed and controlled (foe?) versus the more active role a person is required to play in their health and wellbeing, which might be assisted by technologies (friend?). Yet, very little is known about how this tension is actually experienced.

Whilst critiques of surveillance for health exist, they have tended to examine public health, universalism and global anxieties; the concern has been mass surveillance rather than individuals' and groups' general or technology-specific involvement and understanding. Moreover, these critiques are narrowly conceptualised and focus on informing public health decisions through epidemiology, disease data, medical diagnostics, syndromic surveillance and clinical management:

The continuous, systematic collection, analysis and interpretation of health-related data needed for the planning, implementation, and evaluation of public health practice (World Health Organisation 2014).

Discourses of 'sousveillance' (Mann et al. 2003) have also gained momentum among those who devise their own strategies and practice self-monitoring for their own purposes, for example within the 'Quantified Self' movement. Various self-monitoring/data-generating strategies and technologies are voluntarily devised by individuals and groups, primarily for self-management. These people consider themselves to have substantial autonomy in self-managing their health and wellbeing because they define, measure and adapt it on their own terms. This 'quantified self' does not necessarily interact with the medical model in meaningful ways, if at all. Therefore, the flow of surveillance is not automatically authoritarian or paternalistic since both active and passive roles arise. These can be assumed by either health professionals or individuals and communities, potentially reshaping dynamics of power, responsibility and dimensions of empathy and trust.

Sousveillance has been deliberated, but only in a delocalised clinical gaze through which patients engage with population level techniques in a distributed form, increasingly through popular culture and media. This gaze may facilitate direct impact or meaningfulness. However, it is not without concern, as Day (2009) and Bell (2009) illustrate through their examinations of surveillance/sousveillance in anorexia 
nervosa. Social constructions of anorexia nervosa as a feminised, gendered disease (and so with constructions of other conditions associated with other characteristics) rely on shared cultural norms and inherent epistemic injustice(s) around knowing (Fricker 2007). These may a priori disempower, marginalise and even ignore the individual, disregarding complex negotiations of medical surveillance undertaken by those who are defined, or self-define, as suffering from a disease (Bell 2009).

Furthermore, emphasis remains on dependence rather than autonomy. There is a conflict between external objectification and an internal requirement to partake, which is consequently as constraining. Narrow characterisations neglect broader determinants of health. They disallow individualised or groups' holistic consideration of surveillant technologies' intersection with and integration into everyday health and wellbeing, and even less the means by which individuals might be enabled to live better and/or well. Tensions between oppressive monitoring by others and generating one's own data mean that surveillance for health is insufficiently conceptualised and underexplored-as either friend or foe-especially in relation to social transformations and ethical considerations.

During the Surveillance and/in Everyday Life conference, held in Australia in 2012, pertinent questions about the evolution of surveillance within health and social care contexts were collectively raised. Although the proceedings (French and Smith 2013) focused on public health, the broad applicability of the notion of surveillance was acknowledged. Outlining an agenda for future research, Lupton (2013) identified everyday life as a key site for inquiry into how technologies feature. This is a new perspective for the field that has yet to be adequately theorised or studied in empirical settings. French and Smith (2013) therefore urge that research pays greater critical attention to health and social care surveillance and its complexities. Current ongoing multidisciplinary work at the University of Aberdeen and planned future collaborations on international multidisciplinary research will contribute to this underexplored and important area of surveillance within contemporary society.

\section{Acknowledgements}

With thanks to Professor Lorna McKee, Professor Vikki Entwistle and Dr Steve Turner (all University of Aberdeen, UK), Professor Deborah Lupton (University of Canberra, Australia) and Sara Riggare (Karolinska Institutet, Stockholm, Sweden) for most helpful discussions, their comments on earlier drafts and our ongoing and future collaborations.

\section{References}

Bell, Mebbie. 2009. Prosthetic Surveillance: The medical governance of healthy bodies in cyberspace. Surveillance \& Society 6(2): 151-162.

Day, Cheryl. 2009. Does my bum look big in this? Reconsidering anorexia nervosa within the culture context of 20th century Australia. Surveillance \& Society 6(2):142-150.

Earle, Sarah, Pam Foley, Carol Komaromy and Cathy Lloyd. 2009. Editorial: Health, Medicine and Surveillance in the $21^{\text {st }}$ Century. Surveillance \& Society 6(2): 96-100.

Foucault, Michel. 1973 [1963]. The Birth of the Clinic. New York: Vintage.

French, Martin and Gavin Smith. 2013. Editorial: 'Health' surveillance: new modes of monitoring bodies, populations and polities. Critical Public Health 23(4):383-392.

Fricker, Miranda. 2007. Epistemic Injustice: Power and the Ethics of Knowing. Oxford: Oxford University Press.

Information Commissioner. 2006. Surveillance in Society. Accessed $03 \quad$ March 2014. http://www.parliament.uk/business/publications/research/key-issues-for-the-new-parliament/security-andliberty/surveillance-society/

International Journal of Monitoring and Surveillance Technologies Research. 2013. 1(2-4).

Lupton, Deborah. 2013. Quantifying the body: monitoring and measuring health in the age of mHealth technologies. Critical Public Health 23(4): 393-403.

Lyon, David. 2002. Surveillance Studies: Understanding visibility, mobility and the phonetic fix. Surveillance \& Society 1(1):1-7.

Mann, Steve, Jason Nolan and Barry Wellman. 2003. Sousveillance: Inventing and Using Wearable Computing Devices for Data Collection in Surveillance Environments. Surveillance \& Society 1(3): 331-355.

Morgan, Heather M. 2013. Regulating CCTV?: We can't solve problems by using the same kind of thinking we used when we created them. Critical Criminology 21(1): 15-30.

World Health Organisation. 2014. Accessed 03 March 2014. http:/www.who.int/en/ 